Nuclear Physics A (2001) in press.

\title{
Probing Mechanical and Chemical Instabilities in Neutron-Rich Matter
}

\author{
Bao-An Li*, Andrew T. Sustich, Matt Tilley and Bin Zhang \\ Department of Chemistry and Physics \\ P.O. Box 419, Arkansas State University \\ State University, Arkansas 72467-0419, USA
}

\begin{abstract}
The isospin-dependence of mechanical and chemical instabilities is investigated within a thermal and nuclear transport model using a Skyrme-type phenomenological equation of state for neutron-rich matter. Respective roles of the nuclear mean field and the 2-body stochastic scattering on the evolution of density and isospin fluctuations in either mechanically or chemically unstable regions of neutron-rich matter are investigated. It is found that the mean field dominates overwhelmingly the fast growth of both fluctuations, while the 2-body scattering influences significantly the later growth of the isospin fluctuation only. The magnitude of both fluctuations decreases with the increasing isospin asymmetry because of the larger reduction of the attractive isoscalar mean field by the stronger repuslive neutron symmetry potential in the more neutron-rich matter. Moreover, it is shown that the isospin fractionation happens later, but grows faster in the more neutron-rich matter. Implications of these results to current experiments exploring properties of
\end{abstract}

*email: Bali@astate.edu 
neutron-rich matter are discussed.

PACS numbers: 25.70.-z, 25.75.Ld., 24.10.Lx

Key Words: Isospin physics, equation of state, neutron-rich matter, fluctuations and instabilities 


\section{INTRODUCTION}

Explosion mechanisms of supernova and related properties of neutron stars are among the most interesting topics of modern nuclear astrophysics. Investigations into these questions rely critically on the knowledge about the equation of state of isospin asymmetric nuclear matter. Thus, one of the most important subjects in nuclear physics is the study of the isospin-dependence of the nuclear equation of state using nuclear reactions induced by neutron-rich nuclei or radioactive beams [1 3]. In these reactions transient states of nuclear matter with sufficiently high isospin asymmetries as well as large thermal and compressional excitations can be created. The rapid progress in recent experiments with rare isotopes has therefore made the study of novel properties of extremely isospin-asymmetric nuclear matter possible. The planned Rare Isotope Accelerator (RIA) will further enhance the exploration of this new frontier dramatically [2].

Prospects for discovering new physics in neutron-rich matter have generated much interest in the nuclear science community [3 9]. In particular, the isospin-dependence of the nuclear equation of state (EOS), reflected mainly in the density dependence of the symmetry energy, has recently received much attention. This is because the isospin-dependence of the nuclear EOS is among the most important but very poorly known properties of neutron-rich matter [10 [15]. It is very important to the mechanisms of Type II supernova explosions and neutron-star mergers. It also determines the proton fraction and electron chemical potential in neutron stars at $\beta$ equilibrium. These quantities consequently influence the cooling rate of protoneutron stars and the possibility of kaon condensation in dense stellar matter 16 20. Moreover, the isospin-dependence of the nuclear EOS also determines the stability boundaries of asymmetric nuclear matter. These boundaries and their isospin-dependence subsequently affects the multifragmentation mechanism in nuclear reactions at intermediate energies. Multifragmentation is thought to occur in symmetric nuclear matter due to the growth of instabilities triggered by density fluctuations, such as the Coulomb, surface and volumetric instabilities [21]. While in isospin-asymmetric nuclear matter, it is interesting to 
study not only the isospin-dependence of the above instabilities, but also new mechanisms for nuclear multifragmentation, such as the chemical instability.

In this work we shall explore the isospin-dependence of both mechanical and chemical instabilities using a Skyrme-type phenomenological EOS for neutron-rich matter within both a thermal model and a nuclear transport model. The paper is organized as follows. In Section 2, we shall first outline the isospin-dependent nuclear EOS that we use in this work, and then investigate relevant thermodynamical properties of neutron-rich matter using a thermal model. In Section 3, we shall establish boundaries of the mechanical and chemical instabilities and their isospin-dependence. Then, in Section 4 we study the dynamical growth of mechanical and chemical instabilities and associated phenomena within a nuclear transport model. Finally, a summary and outlook are given in Section 5.

\section{EQUATION OF STATE AND THERMODYNAMICAL PROPERTIES OF NEUTRON-RICH MATTER}

To understand the thermodynamic properties of isospin-asymmetric nuclear matter, we must have a good knowledge of its EOS. The isospin-dependent part of the nuclear EOS is critical for many unique properties of asymmetric matter. At present, nuclear many-body theories predict vastly different isospin-dependent nuclear EOS depending on both the calculation techniques and the bare two-body and/or three-body interactions employed, see e.g., [10 12. Therefore, we use here a Skyrme-type phenomenological EOS for asymmetric nuclear matter. With proper parameterizations, it allows us to sample predictions by different many-body theories and to calculate many thermodynamical properties analytically.

Various studies (e.g., [22,23]) have shown that the energy per nucleon $e(\rho, \delta)$ in asymmetric nuclear matter of density $\rho$ and isospin asymmetry parameter

$$
\delta \equiv\left(\rho_{n}-\rho_{p}\right) /\left(\rho_{n}+\rho_{p}\right)
$$

can be approximated very well by a parabolic function in $\delta$. At zero temperature, the $e(\rho, \delta)$ can be parameterized as 


$$
e(\rho, \delta)=\frac{a}{2}+\frac{b}{1+\sigma} u^{\sigma}+\frac{3}{5} e_{F}^{0} u^{2 / 3}+S(\rho) \cdot \delta^{2}
$$

In the above $u \equiv \rho / \rho_{0}$ is the reduced density, $e_{F}^{0}$ is the Fermi energy and $a=-123.6 \mathrm{MeV}$, $b=70.4 \mathrm{MeV}$ and $\sigma=2$ corresponding to a stiff nuclear EOS of isospin-symmetric nuclear matter. The last term is the symmetry energy whose density dependence is currently rather uncertain [10 15]. We adopt here a form of

$$
S(\rho)=S_{0}\left(\rho_{0}\right) \cdot u^{\gamma}
$$

which was used by Heiselberg and Hjorth-Jensen in their recent studies of neutron stars 24] with $S_{0}\left(\rho_{0}\right)=30 \mathrm{MeV}$ and $\gamma$ as a free parameter. We note here that a value of about $\gamma=0.6$ was obtained by fitting to the result of variational many-body calculations [24,25]. The $\gamma$ parameter is directly related to the $K_{\text {sym }}$ parameter of asymmetric nuclear matter via

$$
\left.K_{\mathrm{sym}} \equiv 9 \rho_{0}^{2} \frac{\partial^{2} S(\rho)}{\partial \rho^{2}}\right|_{\rho=\rho_{0}}=9 S_{0}\left(\rho_{0}\right) \cdot \gamma(\gamma-1)
$$

The values of $K_{\text {sym }}$ predicted by many-body theories scatter from about $-400 \mathrm{MeV}$ to +466 $\mathrm{MeV}$ (e.g. 22]). Experimental values extracted from studying giant monopole resonances of asymmetric nuclei do not constrain the $K_{\text {sym }}$ parameter either. The reported experimental values of $K_{\text {sym }}$ are between $-566 \pm 1350 \mathrm{MeV}$ and $34 \pm 159 \mathrm{MeV}$ [26].

Shown in Fig. 1 are the equations of state with $\gamma=0.5$ and 1 corresponding to $K_{\text {sym }}=$ $-68 \mathrm{MeV}$ and 0 , respectively. The saturation points of asymmetric nuclear matter with different $\delta$ are linked with the dashed lines to guide the eye. These two $\gamma$ parameters lead to rather different saturation points especially for neutron-rich matter. The main features of the equation of state obtained with $\gamma=0.5$ and $\gamma=1$ are surprisingly similarly to those based on the Skyrme Hartree-Fock (SHF) and the Relativistic Mean Field (RMF) models [27, respectively.

It is well known that the symmetry energy has a kinetic and a potential contribution

$$
S(\rho)=\frac{3}{5} e_{\mathrm{F}}^{0} u^{\frac{2}{3}}\left(2^{\frac{2}{3}}-1\right)+V_{2}
$$


where $V_{2}$ is the potential contribution. The corresponding symmetry potential energy density is

$$
W_{a s y}=V_{2} \rho \delta^{2},
$$

and the single-particle potential $v_{\text {asy }}^{q}$ can be obtained from

$$
v_{\text {asy }}^{q}=\frac{\partial W_{a s y}}{\partial \rho_{q}}= \pm\left(S_{0} u^{\gamma}-12.7 u^{2 / 3}\right) \delta+\left(S_{0}(\gamma-1) u^{\gamma}+4.2 u^{\frac{2}{3}}\right) \delta^{2}
$$

where "+" and "" are for $q=$ neutron and $q=$ proton, respectively. Shown in Fig. 2 are the symmetry energy and the corresponding symmetry potential with three $\gamma$ parameters. The magnitude of repulsive (attactive) symmetry potentials for neutrons (protons) increases with both the density $\rho$ and isospin asymmetry $\delta$. The most important effect of $v_{a s y}^{q}$ is to cause the migration of neutrons (protons) from relatively high (low) to low (high) density regions leading to the isospin fractionation (distillation) effect, and this effect grows with increasing $\delta$.

For asymmetric nuclear matter at a finite temperature $T$, the nucleon chemical potential $\mu_{q}$ corresponding to the EOS of Eq. 2 is given by [28]

$$
\mu_{q}=a u+b u^{\sigma}+v_{\mathrm{asy}}^{q}+T\left[\ln \left(\frac{\lambda_{T}^{3} \rho_{q}}{2}\right)+\sum_{n=1}^{\infty} \frac{n+1}{n} b_{n}\left(\frac{\lambda_{T}^{3} \rho_{q}}{2}\right)^{n}\right],
$$

where $\lambda_{T}=\left[2 \pi \hbar^{2} /\left(m_{q} T\right)\right]^{1 / 2}$ is the thermal wavelength of a nucleon. The coefficients $b_{n}$ are obtained from mathematical inversion of the Fermi distribution function [28]. From the above chemical potentials for neutrons and protons, the global pressure for asymmetric nuclear matter can be obtained from the Gibbs-Duhem relation

$$
\frac{\partial P}{\partial \rho}=\frac{\rho}{2}\left[(1+\delta) \frac{\partial \mu_{n}}{\partial \rho}+(1-\delta) \frac{\partial \mu_{p}}{\partial \rho}\right]
$$

We separate the result according to

$$
P=P_{0}+P_{a s y}+P_{k i n}
$$

where $P_{0}$ is the isospin-independent nuclear interaction contribution 


$$
P_{0}=\frac{1}{2} a \rho_{0} u^{2}+b \frac{\sigma \rho_{0}}{\sigma+1} u^{\sigma+1}
$$

$P_{k i n}$ is the kinetic contribution

$$
P_{\text {kin }}=T \rho\left\{1+\frac{1}{2} \sum_{n=1}^{\infty} b_{n}\left(\frac{\lambda_{T}^{3} \rho}{4}\right)^{n}\left[(1+\delta)^{n+1}+(1-\delta)^{n+1}\right]\right\},
$$

and $P_{a s y}$ is the contribution from the isospin-dependent nuclear interaction $V_{2}$

$$
P_{a s y}=\left(S_{0} \gamma \rho_{0} u^{\gamma+1}-8.5 \rho_{0} u^{\frac{5}{3}}\right) \delta^{2}
$$

The total pressure $P$ depends on the isospin asymmetry $\delta$ through both the kinetic pressure $P_{\text {kin }}$ and the asymmetry pressure $P_{a s y}$. We compare the relative values of the three partial pressures in Fig. 3 for a typical temperature of $5 \mathrm{MeV}$ with $\gamma=0.5$ and $\delta=0.2,0.4$ and 0.6, respectively. It is seen that the pressure increases with the increasing isospin asymmetry and $P_{a s y}$ has an appreciable contribution to the total pressure. The isothermal spinodal (ITS) line moves toward lower densities as the isospin asymmetry increases. Fig. 4 shows in more detail the interplay of temperature T, isospin asymmetry $\delta$ and the $\gamma$ parameter. In each window, the isospin asymmetry $\delta$ goes from 0 to 1 (bottom to top) with an increment of 0.2. By increasing the isospin asymmetry the pressure increases as if the temperature is increasing. It is also seen that the $\gamma$ parameter has a small effect on the total pressure especially at high temperature because of the dominating role of the kinetic pressure.

\section{ISOSPIN-DEPENDENCE OF MECHANICAL AND CHEMICAL INSTABILITIES}

Based on the EOS obtained from various microscopic theories and phenomenological models [29 35], it has long been predicted that isospin-asymmetric nuclear matter under certain conditions can be mechanically or chemically unstable, i.e.,

$$
\left(\frac{\partial P}{\partial \rho}\right)_{T, \delta} \leq 0 \quad \text { (mechanical) }
$$

or 


$$
\left(\frac{\partial \mu_{n}}{\partial \delta}\right)_{P, T} \leq 0 \quad \text { (chemical) }
$$

In these unstable regions, small fluctuations in $\rho$ or $\delta$ respectively are expected to grow.

Mechanical instabilities have been well studied in the past for isospin-symmetric systems, here we concentrate on probing its isospin-dependence. Results presented in Fig. 3 and 4 allow us to explore effects of the temperature, density and $\gamma$ parameter on not only the pressure itself, but also how these factors influence the mechanical instability. The latter happens in the region where the slope of the pressure with respect to density is negative, as defined in Eq. 14. From results shown in Fig. 3 and 4, it is easy to relate the increase in temperature with a decreasing probability to observe a mechanical instability. It is seen that the mechanical instability region shrinks when either the temperature or the isospin asymmetry increases. Above a critical temperature $T_{c}$ determined by the condition

$$
\left(\frac{\partial P}{\partial \rho}\right)_{T, \delta}=\left(\frac{\partial^{2} P}{\partial \rho^{2}}\right)_{T, \delta}=0
$$

the pressure increases monotonically with density, and the mechanical instability disappears. To be more quantitatively, we show in Fig. 5, the critical temperature as a function of the isospin asymmetry $\delta$ with a $\gamma$ parameter of 0.5 . It is interesting to note that the critical temperature decreases with increasing isospin asymmetry. Thus one expects to see a shrinking mechanical instability region for increasingly more neutron-rich systems. It is important to stress here that the critical temperature $T_{c}$ discussed above is only associated with the disappearance of mechanical instabilities. For isospin-asymmetric nuclear matter, as we shall discuss in detail in the following, the system can still be chemically unstable against density fluctuations and undergo fragmentation above the critical temperature $T_{c}$.

To study the chemical instability and its isospin dependence we show in Fig. 6 the chemical potential isobars $\mu(T, P, \delta)$ as a function of $\delta$ for neutrons and protons with a parameter $\gamma=0.5$ at a typical temperature of $5 \mathrm{MeV}$ and pressure of $0.005,0.02,0.06,0.12$, 0.2 and $0.8 \mathrm{MeV} / \mathrm{fm}^{3}$, respectively. We note that for corresponding pressures, the isobars for neutrons and protons begin from the same chemical potential at $\delta=0$ as one expects. 
The most interesting feature in this plot is that there exists an envelope of pressures $(0.005$ $\mathrm{MeV} / \mathrm{fm}^{3}<p<0.8 \mathrm{MeV} / \mathrm{fm}^{3}$ ) inside of which chemical instability occurs over a range of $\delta$ where

$$
\left(\frac{\partial \mu_{n}}{\partial \delta}\right) \leq 0
$$

or

$$
\left(\frac{\partial \mu_{p}}{\partial \delta}\right) \geq 0
$$

Inside this region, the system is unstable against isospin fluctuations. For instance, if several neutrons have migrated into a region of chemical instability due to some statistical or dynamical fluctuations in a reaction process, the isospin asymmetry parameter $\delta$ in the region will increase and the energy of that region will decrease because of its lower neutron chemical potential. To minimize the total energy of the system, it is then favorable for the system to have even more neutrons move to the chemically unstable region, thus leading to the further growth of the isospin fluctuation. Results at other temperatures show similar features. However, the boundary of the instability region shrinks as the temperatures $\mathrm{T}$ increase, i.e., for increasing $\mathrm{T}$, chemical instability occurs for much less isospin asymmetric matter. The temperature dependence and its origin will be more clearly illustrated in the following.

Having discussed separately the mechanical and chemical instabilities, we now compare their relative boundaries in the configure space of $(T, \rho, \delta)$. The Gibbs-Duhem relation of Eq. 9 can be used to find boundaries of mechanically unstable regions (ITS: isothermal spinodal) in the $\rho-\delta$ plane for given temperatures. Since the chemical instability condition has to be evaluated at constant pressures, the following Maxwellian relation

$$
\left(\frac{\partial \mu_{n}}{\partial \delta}\right)_{T, P}=\left(\frac{\partial \mu_{n}}{\partial \delta}\right)_{T, \rho}-\left(\frac{\partial \mu_{n}}{\partial \rho}\right)_{T, \delta} \cdot\left(\frac{\partial P}{\partial \rho}\right)_{T, \delta}^{-1} \cdot\left(\frac{\partial P}{\partial \delta}\right)_{T, \rho}
$$

is used to find boundaries of the chemically unstable regions (DS: diffusive spinodal). Shown in Fig. 7 are the boundaries of the mechanical (thick lines) and chemical (think lines) instabilities in the $\rho-\delta$ plane with $\gamma=0.5$ at $T=5$ (lower window), 10 (middle window) 
and $15 \mathrm{MeV}$ (upper window), respectively. It is seen that the diffusive spinodal enveloping the region of mechanical instability extends further out into the plane; the two regions of mechanical and chemical instabilities do no overlap. As the temperature increases, both instabilities become less prominent over a more narrow range of densities at smaller isospin asymmetries. It is thus possible to observe phenomena due to the chemical instability even in reactions using stable nuclei at intermediate energies, which can attain local temperatures up to $20 \mathrm{MeV}$ and isospin asymmetries up to about 0.4 [3]. The above features are independent of the parameters of the EOS and are in good agreement with those based on more microscopic many-body theories 29 31]. In particular, we found that the variation of $\gamma$ parameter has very little effect on the instability boundaries. Therefore, in the following a constant of $\gamma=0.5$ is used. The results shown in Fig. 7 not only provide the motivations but also serve as a guidance for our following transport model simulations.

\section{EVOLUTION OF DENSITY AND ISOSPIN FLUCTUATIONS IN MECHANICALLY OR CHEMICALLY UNSTABLE NEUTRON-RICH MATTER}

Density and isospin fluctuations are expected to grow in the mechanically and/or chemically unstable regions of asymmetric nuclear matter. Information about the respective growth rates of these fluctuations and how they depend on the isospin-asymmtry of nuclear matter is currently still rather rare. This information is vitally important for the structure and stability of both neutron stars and radioactive nuclei as well as mechanisms of nuclear multifragmentation in reactions induced by neutron-rich nuclei. In the following, we study the evolution of fluctuations in isospin-asymmetric nuclear matter initialized in the mechanically or chemically unstable regions within an isospin-dependent transport model IBUU [4.5]. We note that Baran et al have used a similar approach [33] in studying thermodynamical instabilities which can be identified either as a mechanical or chemical instability [34.

The IBUU model uses consistently the isospin-dependent EOS and the corresponding 
potentials as in the thermal model outlined in Section 2. Moreover, scatterings among neutrons and protons are fully isospin-dependent in terms of their total and differential cross sections as well as the Pauli blockings. Nucleons are initialized using a Boltzmann distribution function in a cubic box of length $L_{b o x}$ with periodic boundary conditions. The box is further divided into cells of $1 \mathrm{fm}^{3}$ volume in which the average density $\rho_{\text {cell }}$ and isospin asymmetry $\delta_{\text {cell }}$ are evaluated. We used $10^{4}$ test particles per nucleon in evaluating the $\rho_{\text {cell }}$ and $\delta_{\text {cell }}$.

Shown in Fig. 8 is an illustration of the evolution of a system initialized in the mechanically unstable region with $T_{i}=5 \mathrm{MeV}, \delta_{i}=0.6$ and $\rho_{i}=0.05 \mathrm{fm}^{-3}$. For the purpose of this illustration a small box of $L_{b o x}=10 \mathrm{fm}$ is used. The most interesting feature shown here is the gradually increasing isospin fractionation. This is indicated by the spreading of the initial system into regions with $\rho \leq \rho_{i}$ and $\delta \geq \delta_{i}$ and where $\rho \geq \rho_{i}$ but $\delta \leq \delta_{i}$. The degree of isospin fractionation can be quantified by using the ratio $(N / Z)_{\text {gas }} /(N / Z)_{\text {liquid }}$, where $(N / Z)_{\text {gas }}$ and $(N / Z)_{\text {liquid }}$ is the isospin asymmetry of the low $\left(\rho / \rho_{0} \leq 1 / 8\right)$ and high $\left(\rho / \rho_{0}>1 / 8\right)$ density regions, respectively. Shown in Fig. 9 are the degree of isospin fractionation as a function of time for a system initialized with $T_{i}=5 \mathrm{MeV}$ at a density of $\rho_{i}=0.05$ $\mathrm{fm}^{-3}$. As seen from Fig. 7, the system is mechanically unstable with an isospin asymmetry $\delta$ of 0.2 and 0.6 , while it is chemically unstable with $\delta=0.9$. As one expects from the $\delta$ dependence of the symmetry potential $v_{a s y}$, the isospin fractionation grows faster with the increasing $\delta$. It is also interesting to note that the isospin fractionation happens later as the $\delta_{i}$ increases.

The variations of the density $\rho$ and isospin asymmetry $\delta$ with respect to their initial values can be quantified by using, respectively,

$$
\sigma_{d}(t) \equiv\left(\overline{\rho^{2}}-\rho_{i}^{2}\right)^{1 / 2}
$$

and

$$
\sigma_{\delta}(t) \equiv\left(\overline{\delta^{2}}-\delta_{i}^{2}\right)^{1 / 2}
$$


where the average is over all cells. Shown in Fig. 10 and Fig. 11 are the reduced variation in isospin asymmetry $\sigma_{\delta}(t)-\sigma_{\delta}(0)$ and density $\sigma_{d}(t) / \rho_{i}$ as a function of time for a system initialized at $T_{i}=5 \mathrm{MeV}, \rho_{i}=0.05 \mathrm{fm}^{-3}$ and $\delta_{i}=0.2,0.6$ and 0.9 , respectively. As a reference, results are also shown for a system initialized with $T_{i}=15 \mathrm{MeV}$ and $\delta_{i}=0.9$ with the dash-dot lines (with this initial conditions the system is both mechanically and chemically stable as shown in Fig. 7). As one expects, both the isospin and density fluctuations stay almost constant and there is no isospin fractionation at all for this system. While for a system initialized in the mechanically (chemically) unstable region with $\delta_{i}=0.2$ and 0.6 $\left(\delta_{i}=0.9\right)$, it is interesting to see that both the isospin and density fluctuations grow faster with the decreasing isospin asymmetry $\delta_{i}$. This finding is consistent with that found by Baran et al in ref. [33]. Thus, the more neutron-rich system in either the mechanically or chemically unstable regions is more stable against the growth of both density and isospin fluctuations. This is also responsible for the later start of the isospin fractionation in the more neutron-rich systems as shown in Fig. 9.

Why is the more neutron-rich matter more stable against both the density and isospin fluctuations? In the following we try to answer not only this question, but also explore seeds of fluctuations and their isospin dependence, and investigate which aspects of nuclear dynamics are important in governing the growth of fluctuations. The evolution dynamics of asymmetric nuclear matter is governed by the isospin-dependent nuclear mean filed and stochastic nuclear scatterings. It is necessary to study their respective roles in generating the fluctuations. We start with investigating the role of nucleon-nucleon scatterings. In our approach, first-order effects of 2-body stochastic scatterings are included through the collision integral of the BUU equation. Second-order effects from the explicitly stochastic correction to the collision integral as in the Boltzmann-Langevin model are negelected. Thus, t here are two main seeds of fluctuations in our approach, i.e., the initial numerical fluctuations from the random sampling of the initial state and the later 2-body stochastic nuclear scatterings. Both of them may lead to the growth of fluctuations by propagating through the nuclear mean field in the early and later stages of the evolution, respectively. 
The magnitude of the initial numerical fluctuations in both density and isospin asymmetrty is clearly shown in the upper window of Fig. 8. The seeds of fluctuations due to 2-body stochastic nuclear scatterings are determined by the isospin-dependent collision dynamics in asymmetric nuclear matter. Most importantly, the experimental neutron-neutron cross section, as shown in Fig. 12, is only about 1/3 of that for neutron-proton collisions [36], and also the final state for neutron-neutron scatterings is more strongly Pauli blocked in the more neutron-rich matter. To be more quantitative, we show in Fig. 13 the Pauli blocking rate as a function of time. As the $\delta_{i}$ increases from 0.2 to 0.9 the Pauli blocking rate increases from about $35 \%$ to $50 \%$. Therefore, there are significantly less nucleon-nucleon collisions in more neutron-rich nuclear matter. Shown in Fig. 14 are the average number of possible (before using the Pauli blocking) nucleon-nucleon collisions per nucleon $N_{t r y} / A$ and the successful ones $\left(N_{\text {coll }} / A\right)$ as a function of time. The value of $N_{t r y} / A$ reflects directly the isospin-dependent elementary nucleon-nucleon cross sections shown in Fig. 12. Before about $40 \mathrm{fm} / \mathrm{c}$, there is essentially no collision because of the strong Pauli blocking when the phase space nonuniformity due to the initial fluctuation is still small. Later, nuclear scatterings become important, moreover, they are more frequent with the decreasing isospin asymmetry. With $\delta_{i}=0.2$ each nucleon can make about 4 scatterings up to the time of $100 \mathrm{fm} / \mathrm{c}$, while it can only make about 1 collision with $\delta_{i}=0.9$. Because of the combined effects of both the Pauli blocking and the isospin-dependent elementary cross sections, the number of nuclear scatterings in nuclear matter with $\delta_{i}=0.9$ is consequently only about $1 / 6$ of that with $\delta_{i}=0.2$, contributing to the smaller isospin and density fluctuations in the more neutron-rich matter. To separate the respective roles of the nuclear mean field and the 2-body scatterings, we have performed studies by turnning off the nucleon-nucleon collisions in the model. As shown in the upper window of Fig. 15, the collisional seeds of fluctuations lead to the significant growth of the isospin fluctuation in the later stage of the evolution. However, they have very little effect on the growth of density fluctuations as shown in the lower window. A comparison of the results obtained with and without the collision integral indicates that the growth of fluctuations are overwhelmingly dominated by 
the nuclear mean field. This is in agreement with the analysis based on the linear response theory by Baran et al [33. The observed isospin-dependence of the fluctuations can be understood from the interplay between the attractive isoscalar mean field and the repulsive symmetry potential for neutrons. The symmetry potential, as shown in Eq. 7, is repulsive for neutrons and attractive for protons and their magnitudes increase with the increasing isospin asymmetry. Thus, the resultant attractive mean field is weaker in the more neutronrich matter. Because of the small number of scatterings, particularly in the early stage of the evolution, the growth of fluctuations is thus mainly determined by the strength and sign of the resultant nuclear mean field according to the linear response theory. Based on the latter, the magnitude of both fluctuations can grow larger with the increasing strength of the attractive resultant mean field, and thus also with the decreasing isospin asymmetry $\delta$.

\section{SUMMARY AND OUTLOOK}

In summary, utilizing a phenomenological nuclear equation of state within a thermal model, first we explored the isospin dependence of the chemical and mechanical instabilities in asymmetric nuclear matter. The diffusive spinodal is found to extend further out into the $(\delta-\rho)$ plane than the isothermal spinodal. It is shown that the isospin dependence of the nuclear equation of state plays a key role in determining the thermodynamical properties of asymmetric nuclear matter. We also investigated the evolution of density and isospin fluctuations in mechanically and chemically unstable asymmetric nuclear matter within a nuclear transport model. Both the isospin and density fluctuations are found to decrease with the increasing isospin asymmetry of the system. Respective roles of the nuclear mean field and the 2-body stochastic scattering on the evolution of density and isospin fluctuations in either mechanically or chemically unstable regions of neutron-rich matter are investigated. It is found that the mean field dominates overwhelmingly the fast growth of both fluctuations, while the 2-body scattering influences significantly the later growth of the isospin fluctuation only. We have also shown that the isospin fractionation happens later, but grows much faster 
in the more neutron-rich matter.

It is well known that more neutron-rich systems are less bound and have smaller saturation densities as illustrated by the saturation lines in Fig. 1. However, fluctuations and their growth are also important for determining the final state of a neutron-rich system, such as in the projectile fragmentation in producing exotic beams [37. Our results above indicate that fluctuations actually have a compensating role to the lower binding energy in stabilizing neutron-rich system where fluctuations are smaller and do not grow as fast as in symmetric ones. Moreover, our results on the isospin fractionation accompanying the evolution of fluctuations indicate that the configuration of a more dense, isospin-symmetric region surrounded by a more isospin-asymmetric gas as in halo nuclei is a natural result of the isospin-dependent nuclear dynamics. Furthermore, we expect that the multifragmentation and isospin fractionation in nuclear reactions induced by neutron-rich nuclei to happen on longer time scales compared to symmetric reactions of the same masses. These expectations can be tested by measuring products of multifragmentation. In particular, the measurement of neutron-neutron, proton-proton as well as fragment-fragment correlation functions, in comparative studies of isospin symmetric and asymmetric nuclear reactions will be very useful [38, 39].

\section{ACKNOWLEDGEMENT}

We would like to thank V. Baran, M. Colonna, M. Di Toro, A. Evans, J.B. Natowitz, M.B. Tsang and S.J. Yennello for useful discussions. This work was supported in part by the National Science Foundation Grant No. 0088934 and Arkansas Science and Technology Authority Grant No. 00-B-14. 


\section{REFERENCES}

[1] A. Mueller and B. Sherril, Annu. Rev. Nucl. Part. Sci. 43, 529 (1993); P.G. Hansen, A.S. Jensen and B. Jonson, Ann. Rev. Nucl. Part. Sci. 45, 591 (1995); I. Tanihata, Prog. of Part. and Nucl. Phys., 35 (1995) 505; W. Nazarewicz, B. Sherril, I. Tanihata and P. Van Duppen, Nucl. Phys. News 6, 17 (1996).

[2] RIA Physics White Paper, 2000, Eds. R. Casten, W. Nazarewicz et al..

[3] Isospin Physics in Heavy-Ion Collisions at Intermediate Energies, Eds. Bao-An Li and W. Udo Schröder, ISBN 1-56072-888-4, Nova Science Publishers, Inc (2001, New York).

[4] B.A. Li et al., Phys. Rev. Lett. 76, 4492 (1996); ibid, 78, 1644 (1997); B.A. Li, Phys. Rev. Lett. 85, 4221 (2000); B.A. Li, Nucl. Phys. A681, 434c (2001).

[5] B.A. Li, C.M. Ko and W. Bauer, topical review, Int. Jou. Mod. Phys. E7, 147 (1998).

[6] J. Pan and S. Das Gupta, Phys. Rev. C57, 1839 (1998)

[7] H.S. Xu et al., Phys. Rev. Lett. 85, 716 (2000); M.B. Tsang et al, Phys. Rev. Lett. 86, 5023 (2001).

[8] G.D. Westfall, Nucl., Phys. A681, 343c (2001).

[9] S.J. Yennello, Nucl., Phys. A681, 317c (2001).

[10] R.B. Wiringa, V. Fiks and A. Fabrocini, Phys. Rev. C38, 1010 (1988).

[11] B.A. Brown, Phys. Rev. Lett. 85, 5296 (2000).

[12] C.J. Horowitz et al., Phys.Rev. C63, 025501 (2001).

[13] M. Colonna et al., Phys. Lett. B428, 1 (1998); M. Di Toro et al., Nucl. Phys. A681, 426c (2001).

[14] U. Lombardo and W. Zuo in ref. [3].

[15] I. Bombaci in ref. [3]. 
[16] J.M. Lattimer, C.J. Pethick, M. Prakash and P. Haensel, Phys. Rev. Lett. 66, 2701 (1991).

[17] I. Bombaci, T.T.S. Kuo and U. Lombardo, Phys. Rep. 242, 165 (1994).

[18] K. Sumiyoshi and H. Toki, Astro. Phys. Journal, 422, 700 (1994); K. Sumiyoshi, H. Suzuki and H. Toki, Astronomy and Astrophysics, 303, 475 (1995).

[19] C.-H. Lee, Phys. Rep. 275, 255 (1996).

[20] M. Prakash et al., Phys. Rep. 280, 1 (1997); J. Lattimer and M. Prakash, Phys. Rep. 333, 121 (2000).

[21] L.G. Moretto and G.J. Wozniak, Ann. Rev. Nucl. Part. Sci. 43 (1993) 123.

[22] I. Bombaci and U. Lombardo, Phys. Rev. C44, 1892 (1991).

[23] H. Huber, F. Weber and M..K. Weigel, Phys. Lett. B317, 485 (1993); Phys. Rev. C50, R1287 (1994).

[24] H. Heiselberg and M. Hjorth-Jensen, Phys. Rep. 328, 237 (2000).

[25] A. Akmal and V.R. Pandharipande, Phys. Rev. C56, 2261 (1997); A. Akmal, V.R. Pandharipande and D.C. Ravenhall, Phys. Rev. C58, 1804 (1988).

[26] S. Shlomo and D.H. Youngblood, Phys. Rev. C47, 529 (1993).

[27] K. Oyamatsu, I. Tanihata, Y. Sugahara, K. Sumiyoshi and H. Toki, Nucl. Phys. A634, 3 (1998).

[28] H.R. Jaqaman, Phys. Rev. C39, 169 (1988); ibid C40, 1677 (1989).

[29] J.M. Lattimer and D.G. Ravenhall, Astr. Jour., 223, 314 (1978).

[30] M. Barranco and J. R. Buchler, Phys. Rev. C22, 1729 (1980).

[31] H. Müller and B.D. Serot, Phys. Rev. C 52, 2072 (1995). 
[32] B.A. Li and C.M. Ko, Nuc. Phys. A 618, (1997) 498.

[33] V. Baran, M. Colonna, M. Di Toro and A.B. Larionov, Nucl. Phys. A632, 287 (1998).

[34] V. Baran, M. Colonna, M. Di Toro and V. Greco, Phys. Rev. Lett. 86, 4492 (2001).

[35] D. Catalano, G. Giansiracusa and U. Lombardo, Nucl. Phys. A681, 390c (2001).

[36] G. Alkahzov et al., Nucl. Phys. A280, 365 (1977).

[37] W.A. Friedman, M.B. Tsang, D. Bazin and W.G. Lynch, preprint MSUCL-1167, July 2000 .

[38] R. Ghetti et al., Nucl. Phys. A674, 277 (2000); Phys. Rev. C62, 037603 (2000).

[39] W. Bauer, C.K. Gelbke and S. Pratt, Ann. Rev. Nucl. Part. Sci. 42, 77 (1992). 


\section{FIGURES}

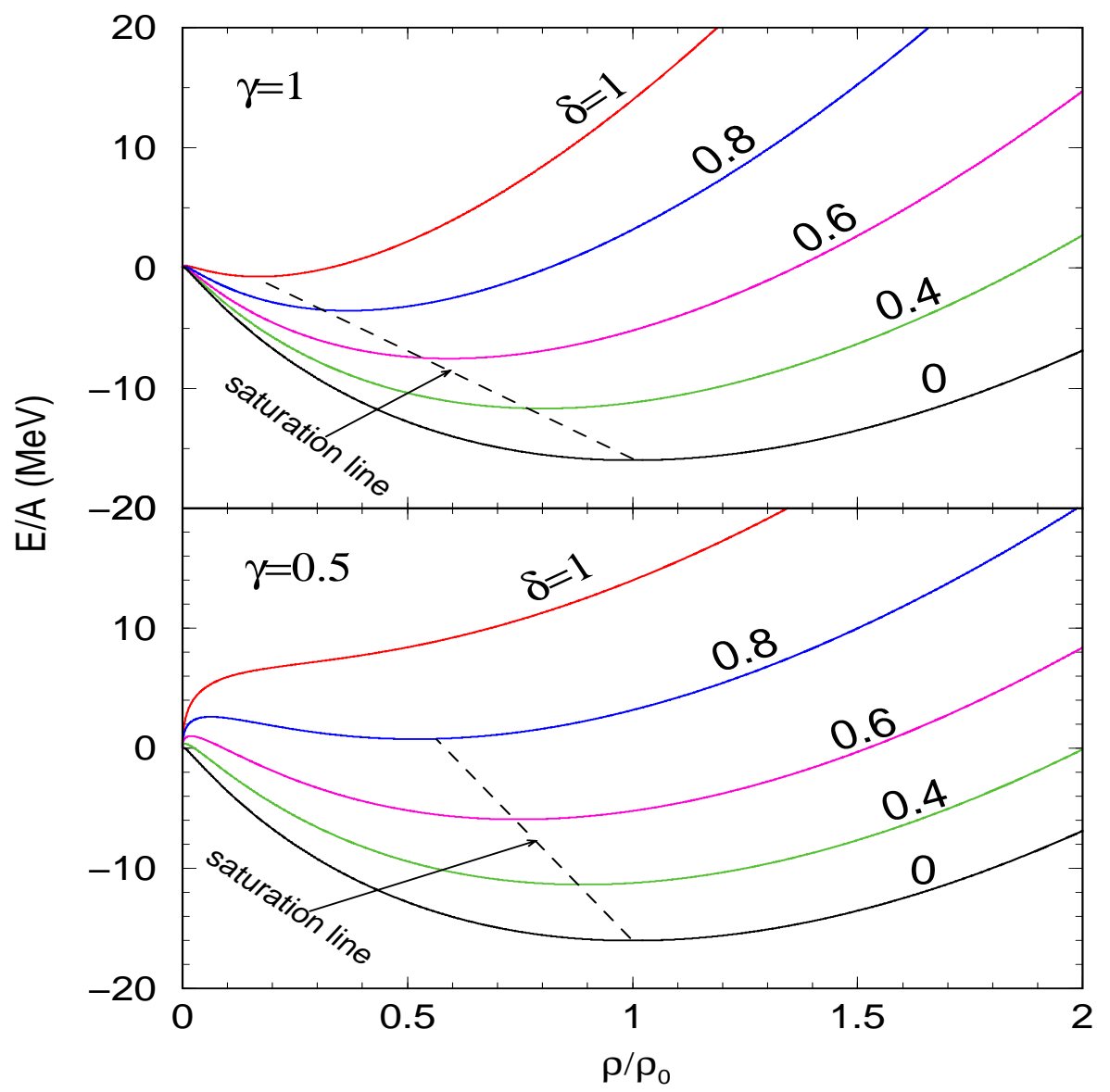

FIG. 1. The equation of state of isospin-asymmetric nuclear matter with the isospin asymmetry $\delta$ spans between 0 and 1 with a $\gamma$ parameter of 0.5 (lower window) and 1 (upper window), respectively. 


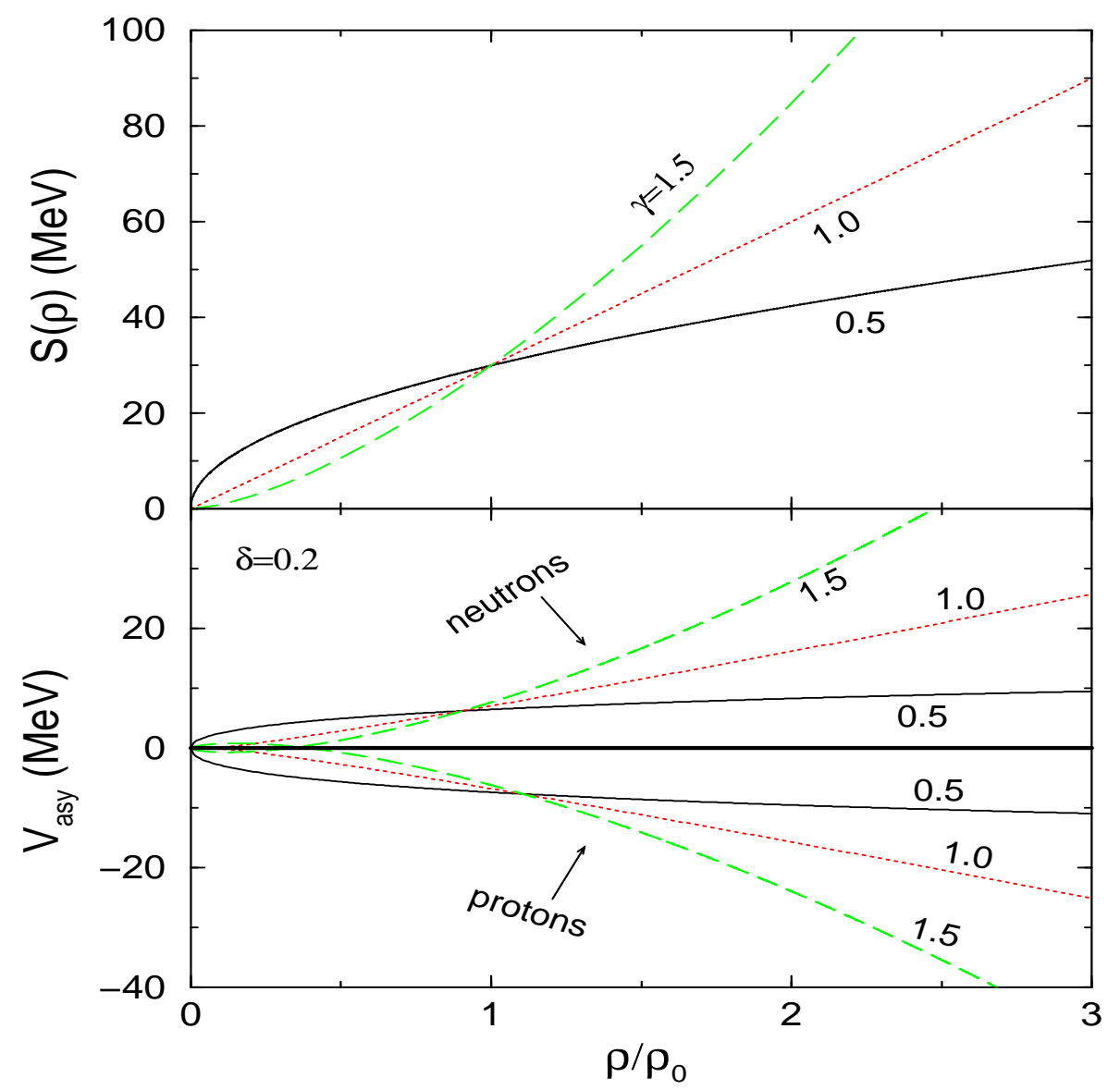

FIG. 2. Symmetry energy (upper window) and potential (lower window) as a function of reduced density with a $\gamma$ parameter of $0.5,1.0$ and 1.5, respectively. The symmetry potential is plotted for an isospin asymmetry $\delta=0.2$. 


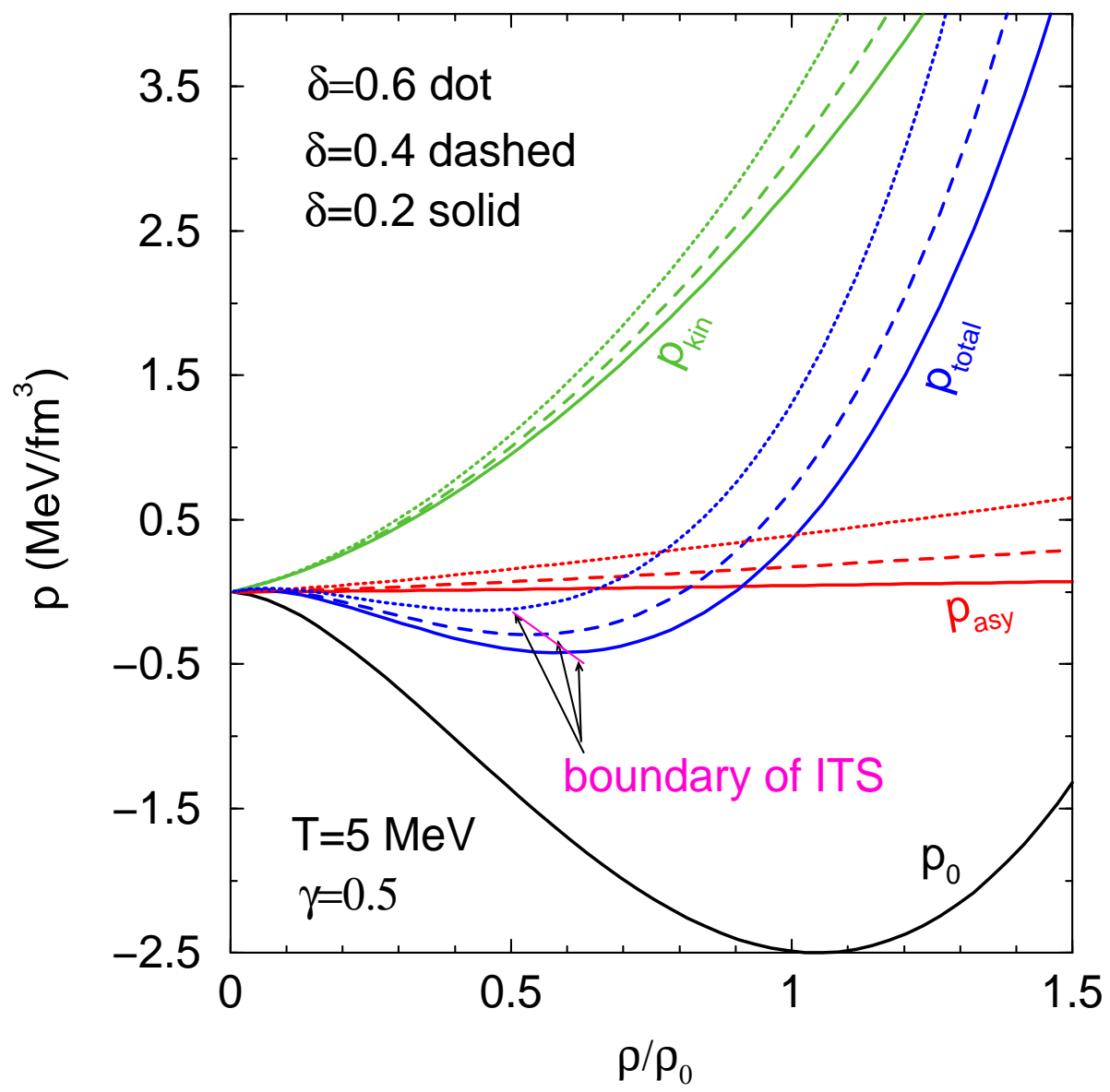

FIG. 3. Total and partial pressures as a function of density, $\rho$, for isospin asymmetry $\delta=0.2,0.4$ and 0.6 with a temperature $T=5 \mathrm{MeV}$ and $\gamma=0.5$. The isothermal spinodal line (ITS) is indicated with arrows. 


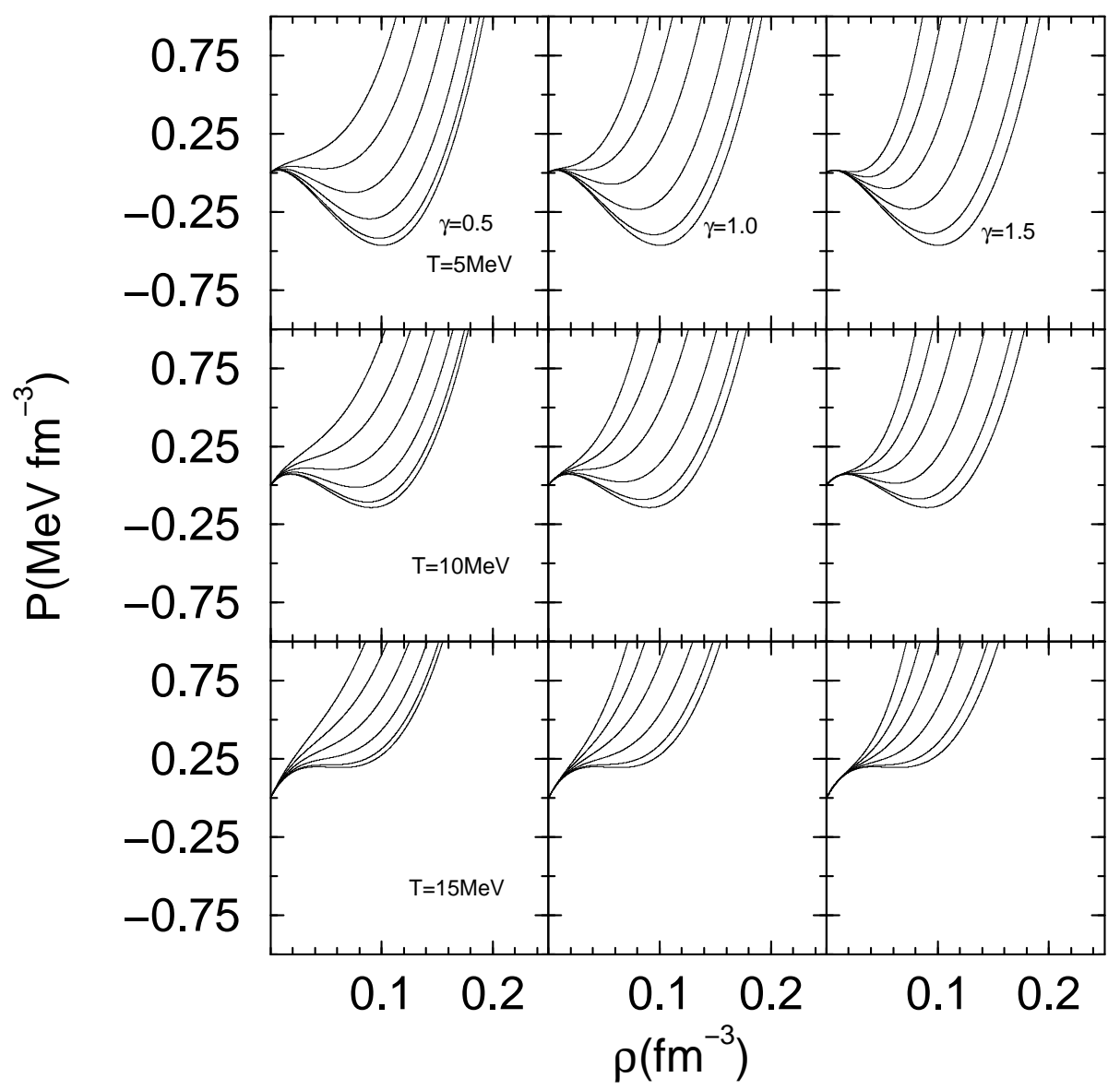

FIG. 4. Total pressure $\mathrm{P}$ as a function of density $\rho$ for isospin asymmetry $\delta=0.0,0.2,0.4,0.6,0.8$ and 1.0 (from bottom to top) with a temperature $T=5,10$ and $15 \mathrm{MeV}$ and $\gamma=0.5,1.0$ and 1.5 , respectively. 


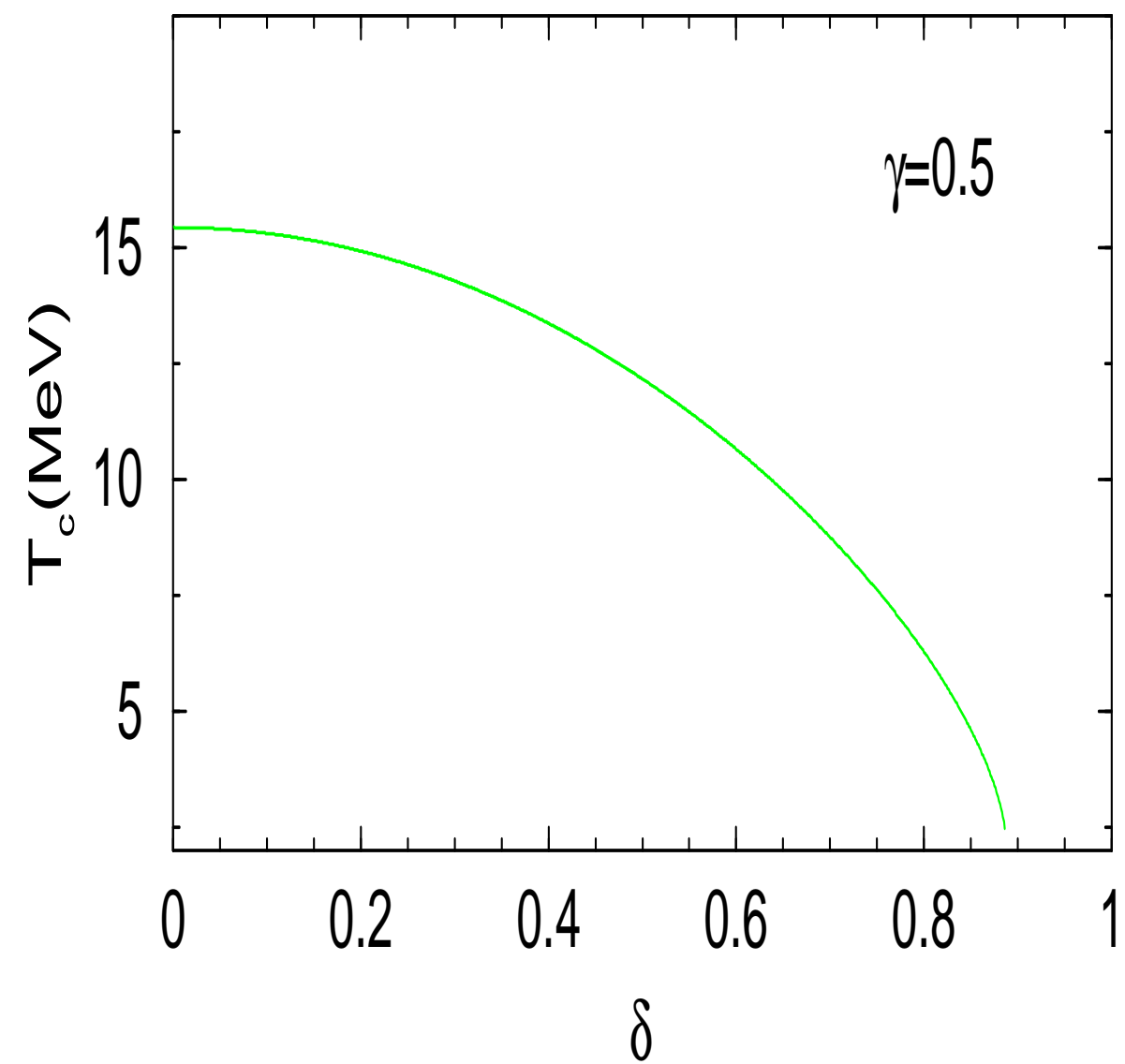

FIG. 5. Critical temperature as a function of isospin asymmetry $\delta$. 


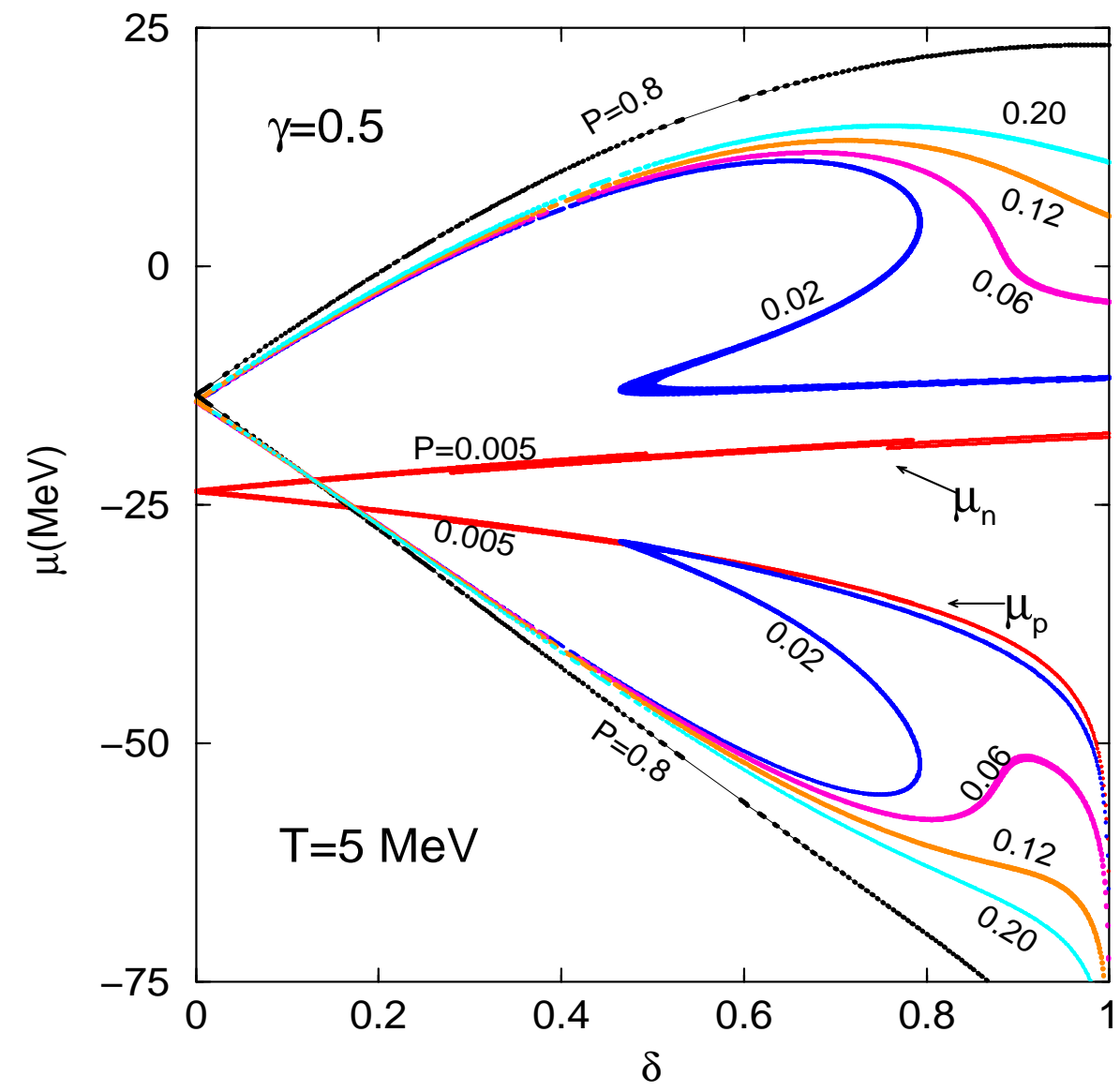

FIG. 6. Chemical potential isobars, $\mu$ for neutrons and protons as a function of isospin asymmetry, $\delta$ at temperature, $\mathrm{T}=5 \mathrm{MeV}$ and density parameter, $\gamma=0.5$. The isobars are at pressures of $P=0.005,0.02,0.06,0.12,0.20$ and $0.80 \mathrm{MeV} / \mathrm{fm}^{3}$, respectively. 


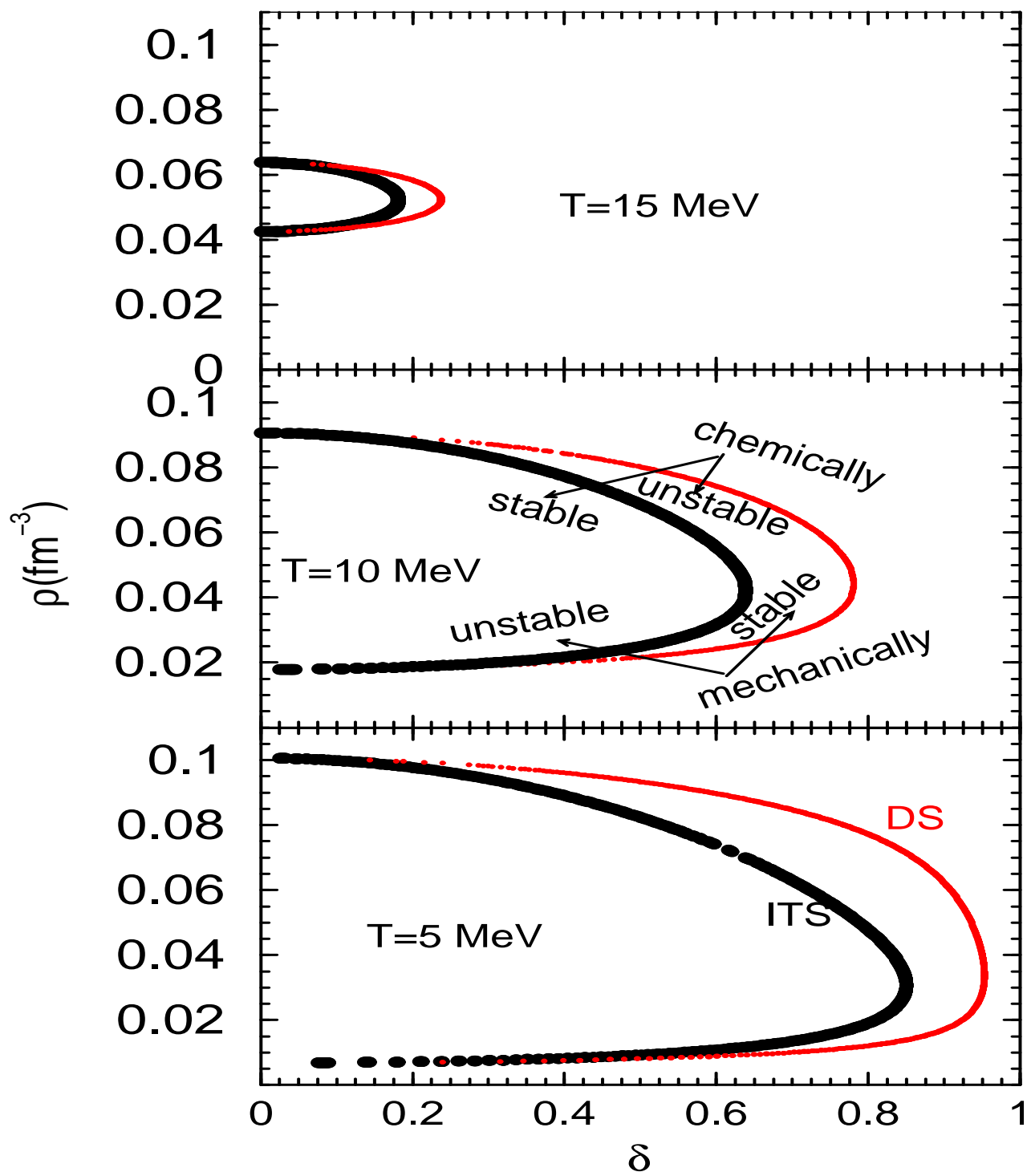

FIG. 7. Boundaries of the mechanical (thick lines) and chemical (thin lines) in the density-isospin asymmetry plane at a temperature of 5, 10 and $15 \mathrm{MeV}$, respectively. 


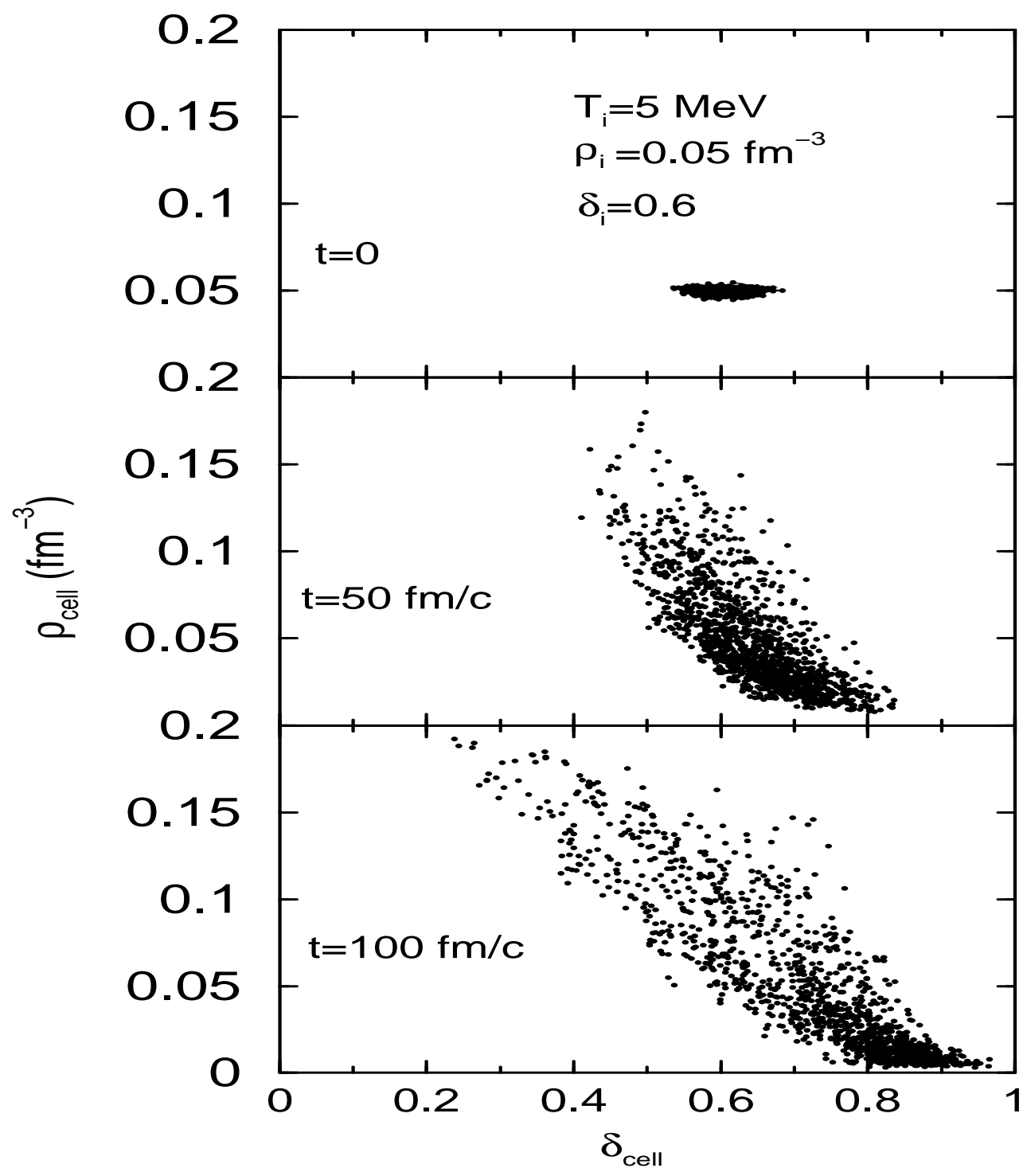

FIG. 8. An illustration of the evolution of asymmetric nuclear matter in the density-isospin asymmetry plane. Each point in the scatter plots represent one cell of $1 \mathrm{fm}^{3}$ volume in a cubic box of side length $L_{b o x}=10 \mathrm{fm}$. 


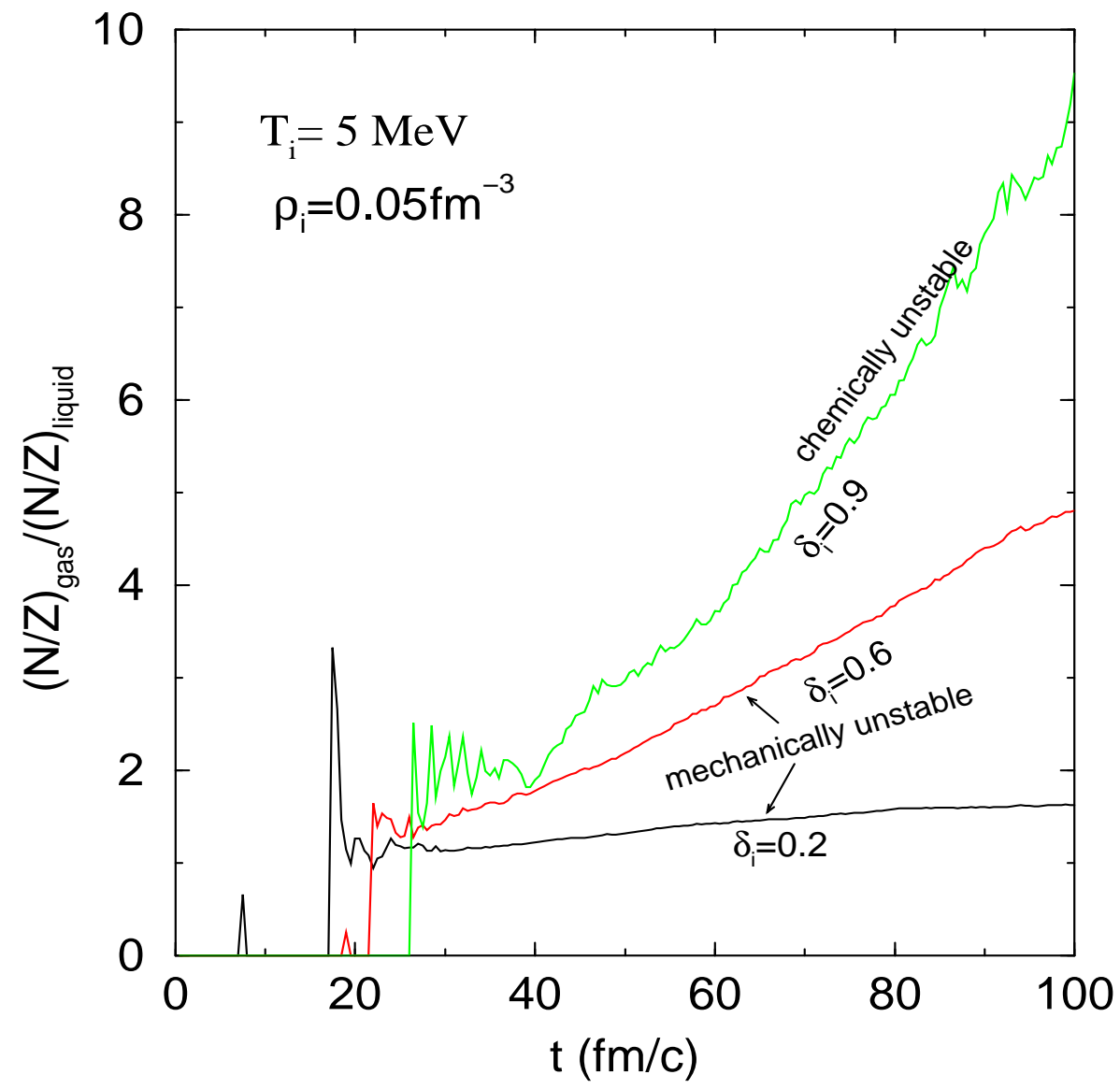

FIG. 9. Evolution of the degree of isospin fractionation in a cubic box of side length $L_{b o x}=30$ fm and density $0.05 \mathrm{fm}^{-3}$, temperature $T_{i}=5 \mathrm{MeV}$ and $\delta_{i}=0.2,0.6$ and 0.9 , respectively. 


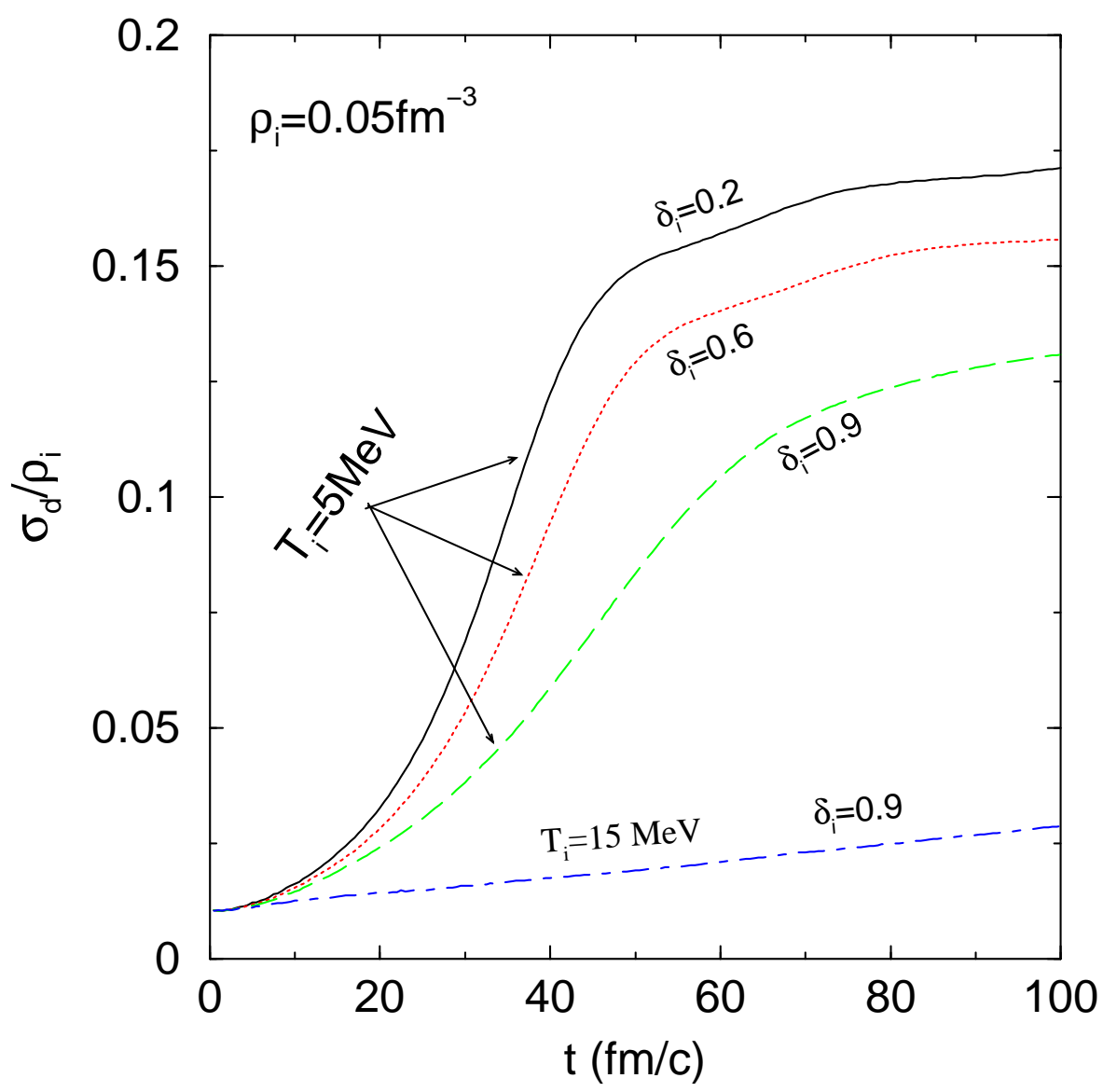

FIG. 10. Evolution of the reduced density fluctuation in a cubic box of side length $L_{b o x}=30$ fm and density $0.05 \mathrm{fm}^{-3}$. The dash-dot lines are calculated with $T_{i}=15 \mathrm{MeV}$ and $\delta_{i}=0.9$; while the solid lines are calculated with $T_{i}=5 \mathrm{MeV}$ and $\delta_{i}=0.2,0.6$ and 0.9 , respectively. 


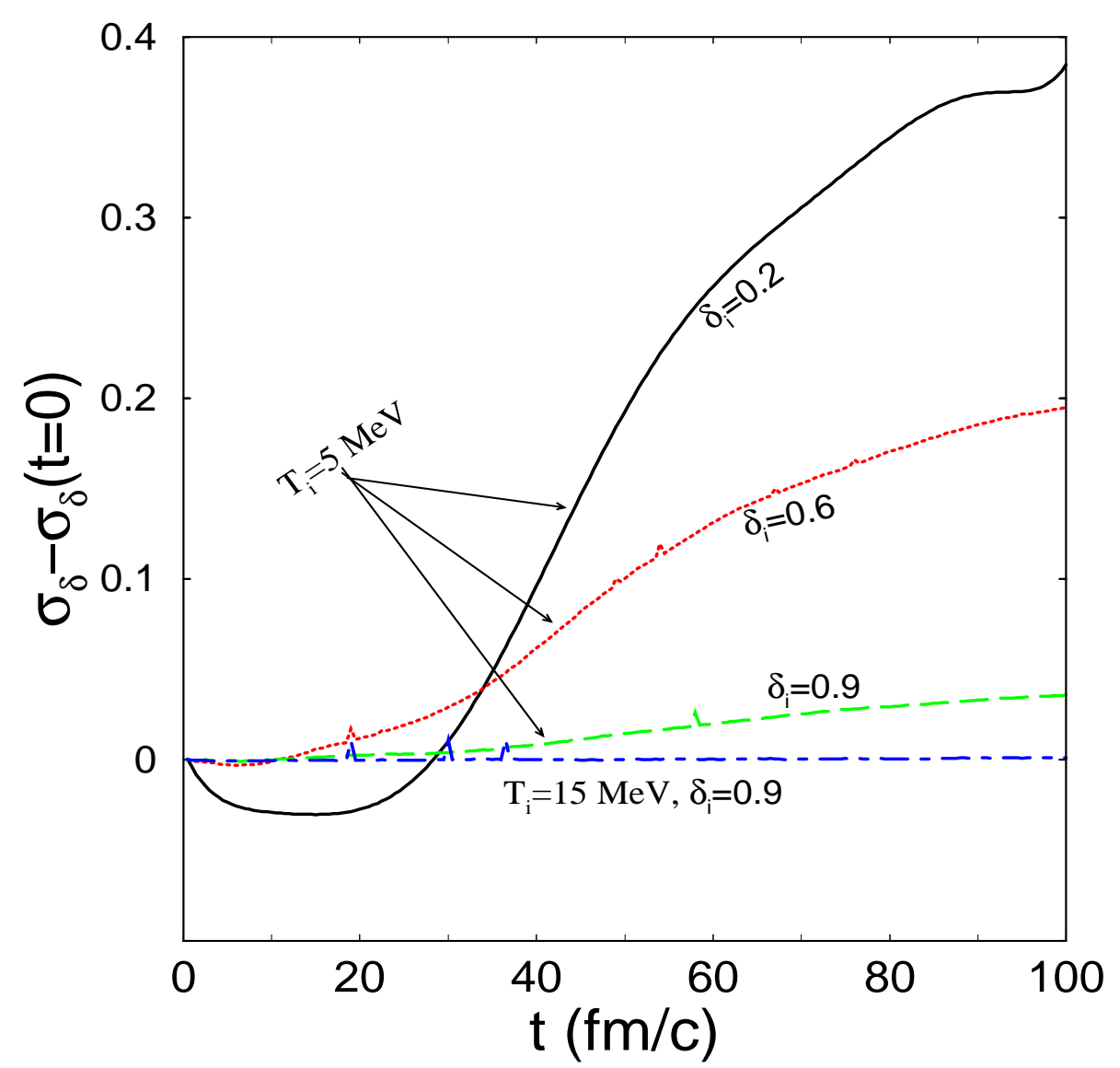

FIG. 11. Evolution of the reduced isospin fluctuation for the same systems as in Fig. 10. 


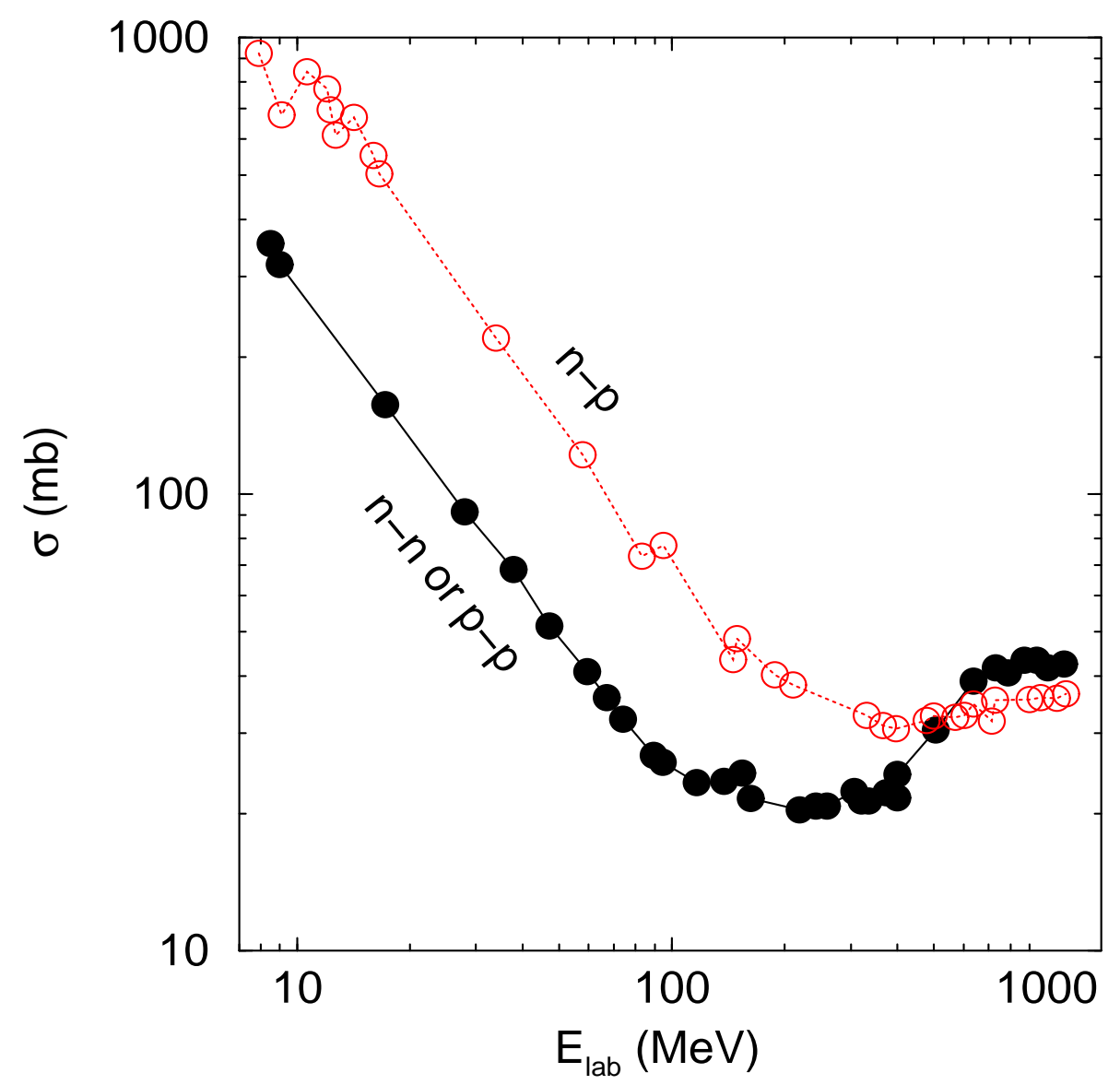

FIG. 12. Experimental neutron-neutron and neutron-proton cross sections as a faction of beam energy. 


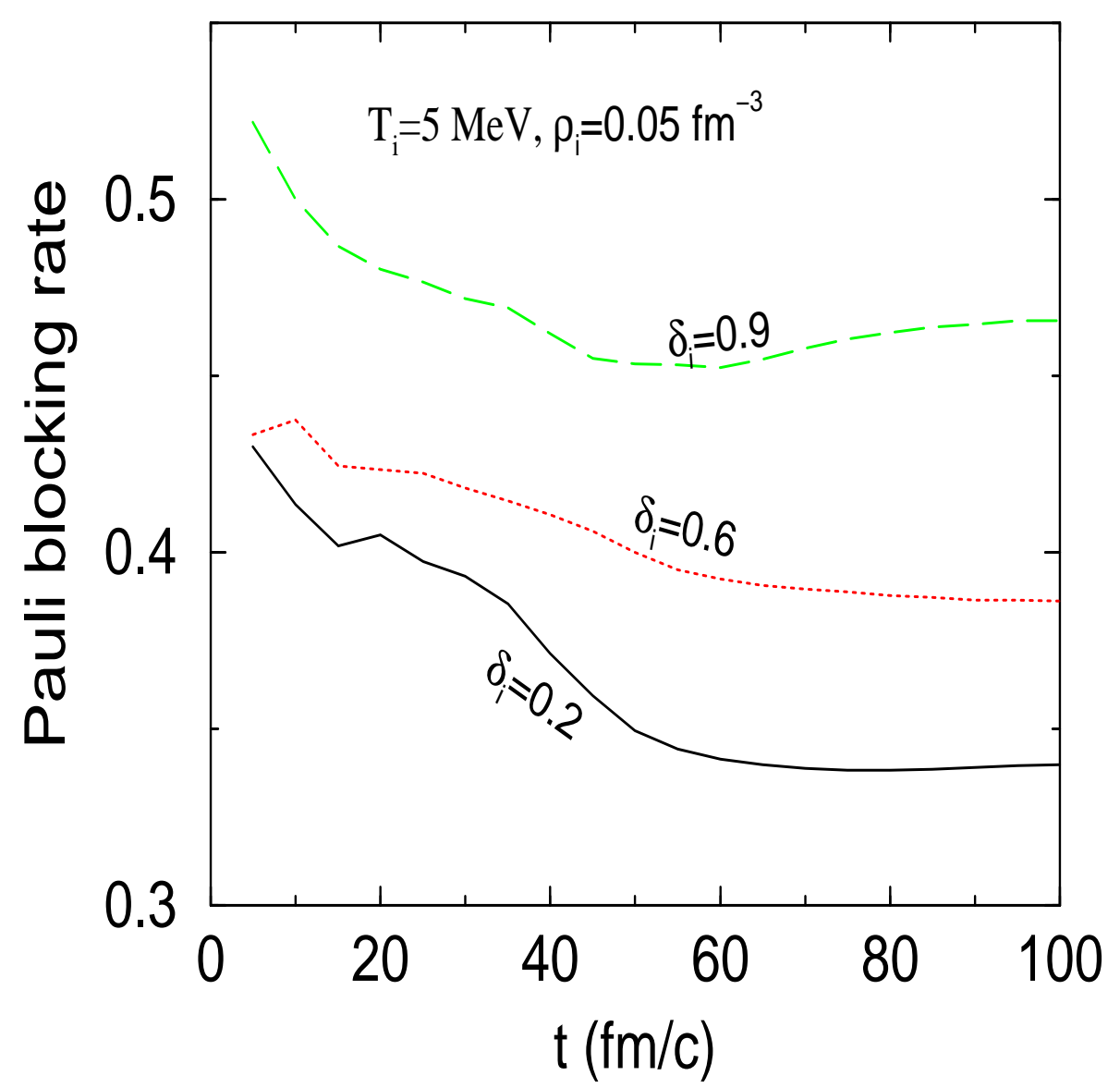

FIG. 13. The Pauli blocking rate as a function of time in a cubic box of $L_{b o x}=30 \mathrm{fm}$ with density $0.05 \mathrm{fm}^{-3}$, temperature $T_{i}=5 \mathrm{MeV}$ and $\delta_{i}=0.2,0.6$ and 0.9 , respectively. 


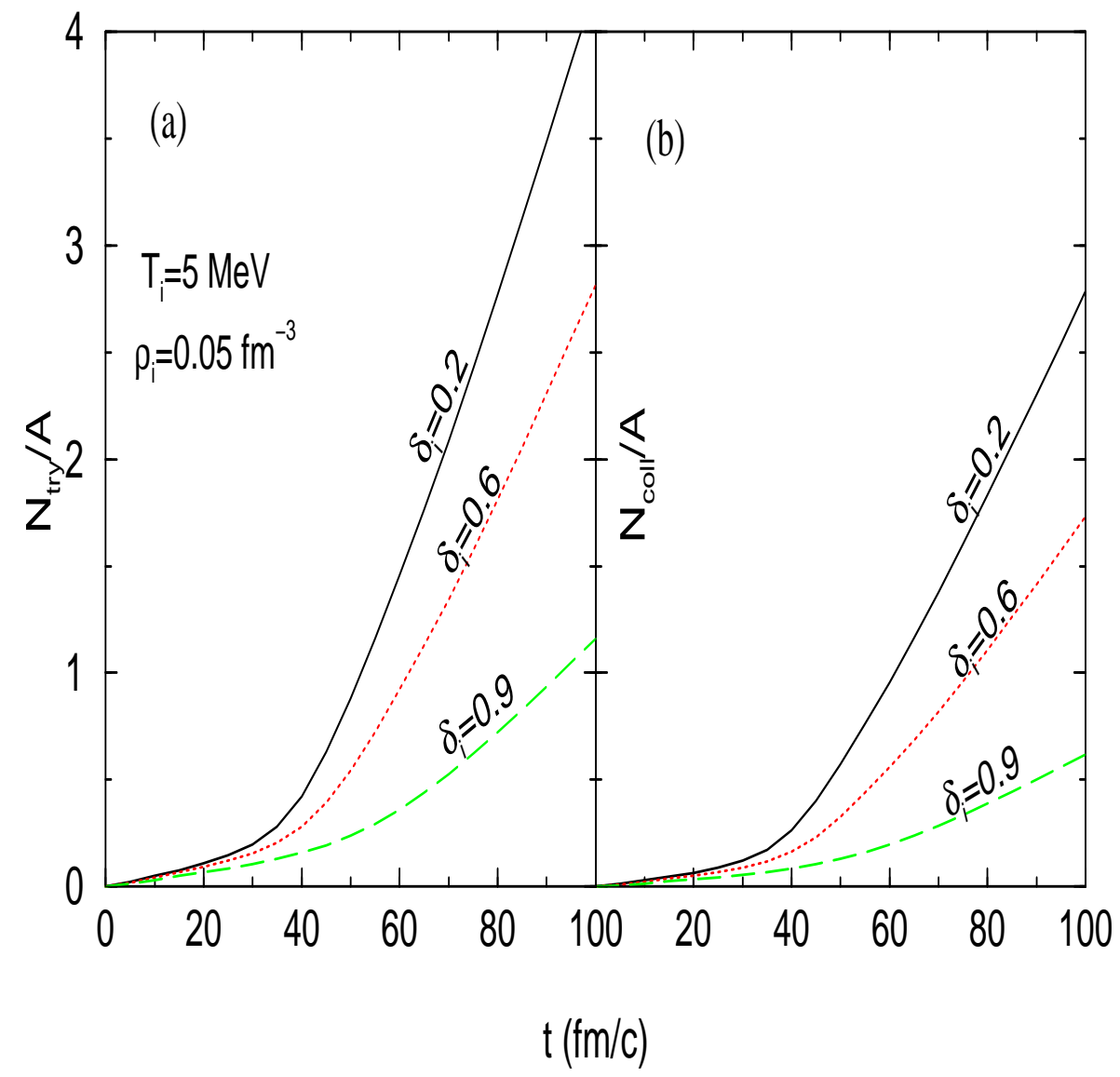

FIG. 14. The average number of possible nucleon-nucleon collisions per nucleon (a) and the average number of successful nucleon-nucleon collisions per nucleon (b) as a function of time for the same systems as in Fig. 13. 


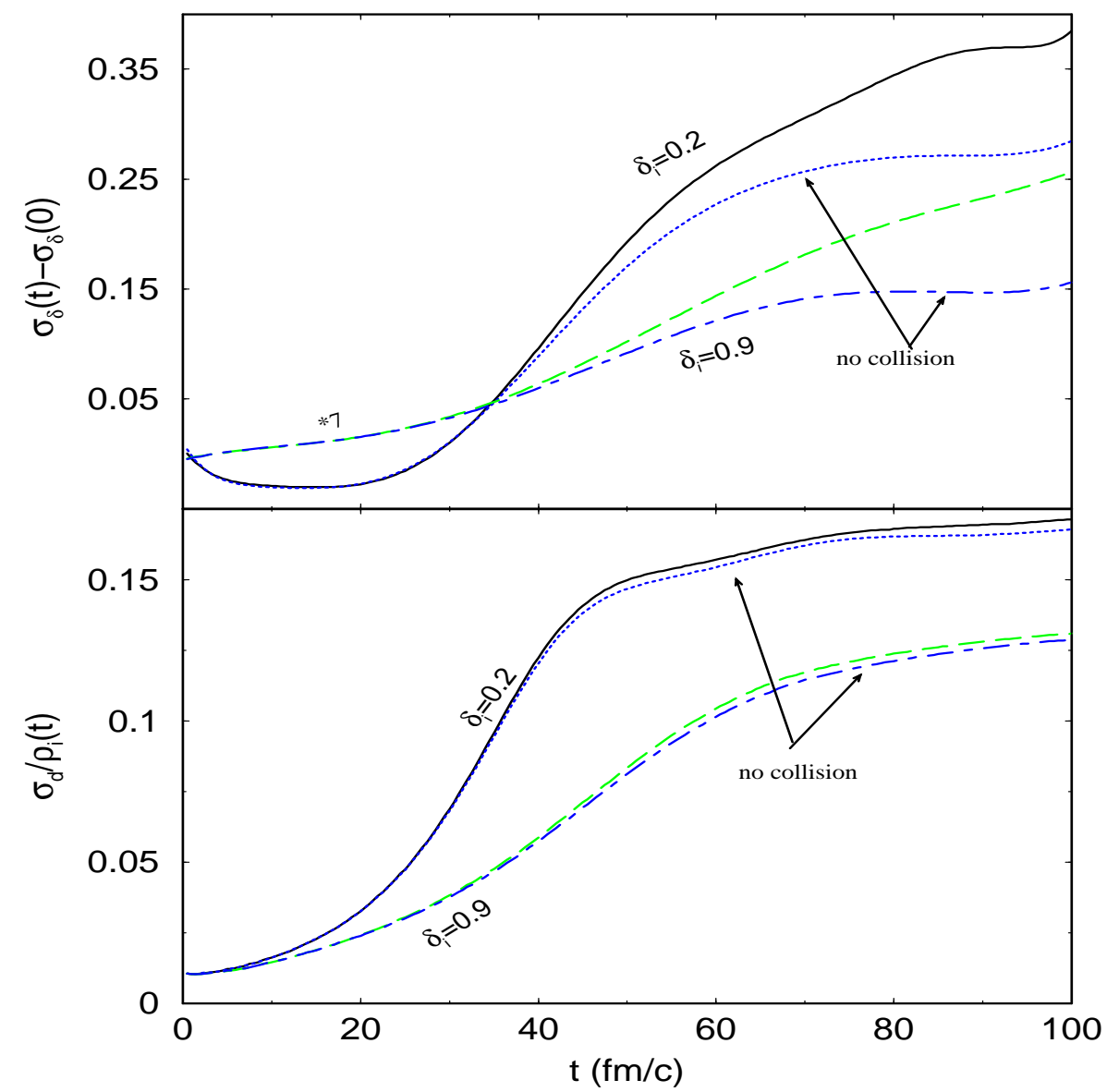

FIG. 15. Evolution of isospin (upper window) and density (lower window) fluctuations as a function of time. The solid and long dashed lines are results of full calculations, while the dotted and dash-dot lines are results obtained by turnning off the 2-body scatterings. 